\title{
Boundary Value Problems of Thermoelasticity for Porous Sphere and for A Space with Spherical Cavity
}

\author{
Lamara Bitsadze*(D) \\ I. Vekua Institute of Applied Mathematics of IV. Javakhishvili Tbilisi State University, University St., 0186 Tbilisi, Georgia.
}

\section{Keywords}

Coupled theory of thermoelasticity for porous materials,

Explicit solution,

Porous sphere,

Space with a spherical cavity.

\begin{abstract}
This article is concerned with the coupled linear quasi-static theory of thermoelasticity for porous materials under local thermal equilibrium. The system of equations is based on the constitutive equations, Darcy's law of the flow of a fluid through a porous medium, Fourier's law of heat conduction, the equations of equilibrium, fluid mass conservation and heat transfer. The system of governing equations is expressed in terms of displacement vector field, the change of volume fraction of pores, the change of fluid pressure in pore network and the variation of temperature of porous material. The present paper is devoted to construct explicit solutions of the quasi-static boundary value problems (BVPs) of coupled theory of thermoelasticity for a porous elastic sphere and for a space with a spherical cavity. In this research the regular solution of the system of equations for an isotropic porous material is constructed by means of the elementary (harmonic, bi-harmonic and metaharmonic) functions. The basic boundary value problems (the Dirichlet type boundary value problem for a sphere and the Neumann type boundary value problem for a space with a spherical cavity) are solved explicitly. The obtained solutions are given by means of the harmonic, bi-harmonic and meta-harmonic functions. For the harmonic functions the Poisson type formulas are obtained. The bi-harmonic and meta-harmonic functions are presented as absolutely and uniformly convergent series.
\end{abstract}

\section{Introduction}

In most solids there are pores through which the liquid or gas may flow. Many materials are known as porous materials, the human skin has a larger number of pores, cancellous bone is considered as a porous material, etc.

The foundations of the theory of elastic materials with voids were first proposed by Cowin and Nunziato $(1979,1983)[1,2]$. They investigated the linear and nonlinear theories of elastic materials with voids. In these theories the independent variables are displacement vector field and the change of volume fraction of pores. Such materials include, rocks and soils, granulated and some other manufactured porous materials. The history of development of porous body mechanics, the main results and the sphere of their application are set forth in detail in the monographs (de Boer 2000, Straughan 2008,2017, Svanadze 2019) [3-6] (see references therein). The generalization of the theory of elasticity and thermoelasticity for materials with double voids belongs to Ieşan and Quintanilla (2014) [7]. In (2020) [8] Svanadze considered the coupled linear model of porous elastic solids by combining the following three variables: the displacement vector field, the volume fraction of pores and the change of fluid pressure in pore network. In this work the basic internal and external BVPs of steady vibrations are investigated, the uniqueness and the existence theorems are proved by means of the potential method and the theory of singular integral equations. The coupled linear theory of thermoelasticity for isotropic porous materials by using the concept of Darcy's law and the volume fraction of pore network are presented by Svanadze (2019) [9]. The quasi-static BVPs of the theories of elasticity and thermoelasticity for porous materials are studied by Mikelashvili $(2020,2021)[10,11]$. These works [8-11] represents a first step in the coupled linear theory of elasticity and thermoelasticity of porous materials.
For applications, it is especially important to construct the solutions of BVPs in explicit form because such solutions enable us to effectively perform quantitative analysis of the investigated problems. Questions related to this topic, different types of problems in the theory of elasticity and thermoelasticity of porous materials are considered, for example, in the works [12-26], where the explicit solutions are constructed for some BVPs for the concrete domains.

The present paper is devoted to construct explicit solutions of the quasi-static boundary value problems (BVPs) of coupled theory of thermoelasticity for porous elastic sphere and for a space with a spherical cavity. The regular solution of the system of equations for an isotropic porous material is constructed by means of the elementary (harmonic, bi-harmonic and meta-harmonic) functions. The basic BVPs for a sphere and for a space with a spherical cavity are solved explicitly.

\section{Basic equations and boundary value problems}

Let $x=\left(x_{1}, x_{2}, x_{3}\right)$ be a point in the Euclidean three-dimensional space $E^{3}$. We consider an isotropic thermoelastic porous sphere $D$, bounded by a surface $S$, with center at the origin and radius $R$. Let $D^{-}$be the whole space with a spherical cavity and with boundary $S$.

The basic homogeneous system of equations in the coupled linear quasi-static theory of thermoelasticity for porous materials expressed in terms of the displacement vector $\mathbf{u}(\mathbf{x})$, the change of the volume fraction $\varphi$, the change of fluid pressure in pore network $p$ and the variation of temperature $\theta$ has the following form (Mikelashvili [11]) 
$\mu \Delta \mathbf{u}+(\lambda+\mu) \operatorname{graddiv} \mathbf{u}+\operatorname{grad}\left(b \varphi-\beta p-\gamma_{0} \theta\right)=0$,

$\left(\alpha \Delta-\alpha_{1}\right) \varphi-b \operatorname{div} \mathbf{u}+m p+\gamma_{1} \theta=0$,

$\left(k \Delta+i \omega a_{0}\right) p+i \omega\left(\beta \operatorname{div} \mathbf{u}+m \varphi+\gamma_{2} \theta\right)=0$,

$\left(k_{0} \Delta+i \omega a T_{0}\right) \theta+i \omega T_{0}\left(\gamma_{0} \operatorname{div} \mathbf{u}+\gamma_{1} \varphi+\gamma_{2} p\right)=0$,

where $\gamma_{0}$ is the thermal expansion coefficient, $a_{0}$ is measures the compressibility of pores, $\alpha, \alpha_{1}, b, m, \gamma_{1}, \gamma_{2}$ are the constitutive coefficients, $k=\frac{k^{\prime}}{\mu^{\prime}}, \mu^{\prime}$ is the fluid viscosity, $k^{\prime}$ is the macroscopic intrinsic permeability associated with the pore network, $k_{0}$ is the thermal conductivity of the porous material, $a$ is the heat capacity, $\lambda, \quad \mu$ are the Lame constants, $\beta$ is the effective stress parameter, $\omega$ is the oscillation frequency, $\omega>0$,

$\Delta$ is the 3D Laplace operator.

Definition 1. A vector-function $\mathbf{U}=(\mathbf{u}, \varphi, p, \theta)^{T}$ defined in the domain $D\left(D^{-}\right)$is called regular if

$\mathbf{U}(\mathbf{x}) \in C^{2}(D) \cap C^{1}(\bar{D})\left(\mathbf{U}(\mathbf{x}) \in C^{2}\left(D^{-}\right) \cap C^{1}\left(\overline{D^{-}}\right)\right)$and the following conditions at infinity are added:

$\mathbf{U} \in O\left(|\mathbf{x}|^{-1}\right), \quad \frac{\partial \mathbf{U}}{\partial x_{j}} \in O\left(|\mathbf{x}|^{-2}\right)$,

$j=1,2,3, \quad|\mathbf{x}|^{2}=x_{1}^{2}+x_{2}^{2}+x_{3}^{2}$.

For the system (1), we pose the following boundary value problems:

Problem 1: (The Dirichlet type BVP) Find a regular solution $\mathbf{U}$, satisfying in $D$ the system of equations (1), if on the boundary $S$ the following conditions are given:

$\mathbf{u}^{+}=\mathbf{f}^{+}(\mathbf{z}), \quad \varphi^{+}=f_{4}^{+}(\mathbf{z}), \quad p^{+}=f_{5}^{+}(\mathbf{z})$,

$\theta^{+}=f_{6}, \quad z \in S$,

Problem 2: (The Neumann type BVP)Find a regular solution $\mathbf{U}$, satisfying in $D^{-}$the system of equations (1), if on the boundary $S$ the following conditions are given:

$\left(\mathbf{P}\left(\partial_{x}, \mathbf{n}\right) \mathbf{u}\right)^{-}=\mathbf{f}^{-}(\mathbf{z}), \quad\left(\frac{\partial \varphi}{\partial n}\right)^{-}=f_{4}^{-}(\mathbf{z})$,

$\left(\frac{\partial p}{\partial n}\right)^{-}=f_{5}^{-}(\mathbf{z}), \quad\left(\frac{\partial \theta}{\partial n}\right)^{--}=f_{6}, \quad z \in S$,

where $\mathbf{f}^{ \pm}=\left(f_{1}, f_{2}, f_{3}\right), \quad f_{4}^{ \pm}, \quad f_{5}^{ \pm}, \quad f_{6}^{ \pm}$are the given functions. Moreover, we assume that $f_{i}^{ \pm}$can be presented in the form of series, $(\cdot)^{ \pm}$denotes the limiting value from $D^{ \pm}$,
$\mathbf{U}^{+}(\mathbf{z})=\lim _{D^{+} \supset x \rightarrow z \in S} \mathbf{U}(\mathbf{x}), \mathbf{U}^{-}(\mathbf{z})=\lim _{D^{-} \supset x \rightarrow z \in S} \mathbf{U}(\mathbf{x})$,

the vector $\mathbf{P}\left(\partial_{x}, \mathbf{n}\right) \mathbf{u}$ is defined in the following form

$\mathbf{P}\left(\partial_{x}, \mathbf{n}\right) \mathbf{u}=\mathbf{T}\left(\partial_{x}, \mathbf{n}\right) \mathbf{u}+\mathbf{n}\left(b \varphi-\beta p-\gamma_{0} \theta\right)$,

$\mathbf{T}\left(\partial_{x}, \mathbf{n}\right) \mathbf{u}$ is the stress vector in the classical theory of elasticity $\mathbf{T}\left(\partial_{x}, \mathbf{n}\right) \mathbf{u}=2 \mu \frac{\partial \mathbf{u}}{\partial n}+\lambda \mathbf{n} \operatorname{div} \mathbf{u}+\mu[\mathbf{n} \cdot \operatorname{rot} \mathbf{u}]$,

$\mathbf{n}$ is the external normal vector on $S$ at $\mathbf{Z} \in S$.

The following assertion holds (for details see Mikelashvili [11]).

Theorem 1. The Problem 1( Problem 2) has one regular solution in $D\left(D^{-}\right)$.

The purpose of this paper is to construct an explicit solution of system (1) for a porous sphere and for a porous space with a spherical cavity.

\section{A representation of regular solution}

The following theorems holds:

Theorem 2. If $\mathbf{U}(\mathbf{u}, \varphi, p, \theta)$ is a regular solution of the system (1) then the functions $\mathbf{u}, \operatorname{div} \mathbf{u}, \varphi, p$ and $\theta$ satisfy the following equations:

$\Delta \Delta\left(\Delta+\lambda_{1}^{2}\right)\left(\Delta+\lambda_{2}^{2}\right)\left(\Delta+\lambda_{3}^{2}\right) \mathbf{u}=0$,

$\Delta\left(\Delta+\lambda_{1}^{2}\right)\left(\Delta+\lambda_{2}^{2}\right)\left(\Delta+\lambda_{3}^{2}\right) \Phi=0$,

where $\lambda_{j}^{2}, \quad j=1,2,3$, are roots of the third-degree algebraic equation(see below), $\boldsymbol{\Phi}=(\operatorname{div} \mathbf{u}, \varphi, p, \theta)$.

Proof. Let $\mathbf{U}$ be a regular solution of the equation (1). Applying the divergence operator to Eq. $(1)_{1}$, we obtain

$\mu_{0} \Delta \operatorname{div} \mathbf{u}+\Delta\left(b \varphi-\beta p-\gamma_{0} \theta\right)=0$,

$\left(\alpha \Delta-\alpha_{1}\right) \varphi-b \operatorname{div} \mathbf{u}+m p+\gamma_{1} \theta=0$,

$\left(k \Delta+i \omega a_{0}\right) p+i \omega\left(\beta \operatorname{div} \mathbf{u}+m \varphi+\gamma_{2} \theta\right)=0$,

$\left(k_{0} \Delta+i \omega a T_{0}\right) \theta+i \omega T\left(\gamma_{0} \operatorname{div} \mathbf{u}+\gamma_{1} \varphi+\gamma_{2} p\right)=0$,

$\mu_{0}=\lambda+2 \mu$.

Let us rewrite the system (3) as follows

$D(\Delta) \boldsymbol{\Phi}=$

$\left(\begin{array}{cccc}\mu_{0} \Delta & b \Delta & -\beta \Delta & -\gamma_{0} \Delta \\ -b & \alpha \Delta-\alpha_{1} & m & \gamma_{1} \\ i \omega \beta & i \omega m & k \Delta+i \omega a_{0} & i \omega \gamma_{2} \\ i \omega T_{0} \gamma_{0} & i \omega T_{0} \gamma_{1} & i \omega T_{0} \gamma_{2} & k_{0} \Delta+i \omega a T_{0}\end{array}\right) \boldsymbol{\Phi}=0$. 
As we will see

$\operatorname{det} D(\Delta)=\alpha \mu_{0} k_{0} k \Delta\left(\Delta+\lambda_{1}^{2}\right)\left(\Delta+\lambda_{2}^{2}\right)\left(\Delta+\lambda_{3}^{2}\right)$,

where $\lambda_{j}^{2}, \quad j=1,2,3$ are roots of third-degree algebraic equation with respect to $\xi$

$\alpha \mu_{0} k_{0} k \xi^{3}-d_{1} \xi^{2}+i \omega d_{2} \xi-(i \omega)^{2} T_{0} d_{3}=0$,

$d_{1}=\mu_{0}\left[-\alpha_{1} k k_{0}+i \omega \alpha\left(a k T_{0}+a_{0} k_{0}\right)\right]$

$+i \omega \alpha\left(k_{0} \beta^{2}+k \gamma_{0}^{2} T_{0}\right)+b^{2} k k_{0}$,

$d_{2}=-\mu_{0} k_{0}\left(\alpha_{1} a_{0}+m^{2}\right)-i \omega \alpha T_{0} \mu_{0}\left(\gamma_{2}^{2}-a a_{0}\right)$

$-\mu_{0} T_{0} k\left(a \alpha_{1}+\gamma_{1}^{2}\right)+$

$+k_{0}\left(a_{0} b^{2}-\alpha_{1} \beta^{2}+2 b m \beta\right)+T_{0} k\left(a b^{2}+2 \gamma_{0} \gamma_{1} b-\gamma_{0}^{2} \alpha_{1}\right)$

$+T_{0} i \omega \alpha\left(a \beta^{2}-2 \gamma_{0} \gamma_{2} \beta+a_{0} \gamma_{0}^{2}\right)$,

$d_{3}=\mu_{0}\left(\alpha_{1} \gamma_{2}^{2}-a_{0} \gamma_{1}^{2}+2 m \gamma_{1} \gamma_{2}\right)-\mu_{0} a\left(\alpha_{1} a_{0}+m^{2}\right)$

$+a\left[a_{0} b^{2}-\alpha_{1} \beta^{2}+2 b m \beta\right]+2 \gamma_{0} \gamma_{1}\left(a_{0} b+2 m \beta\right)$

$+2 \alpha_{1} \beta \gamma_{0} \gamma_{2}-a_{0} \alpha_{1} \gamma_{0}^{2}-\left(m \gamma_{0}+b \gamma_{2}+\beta \gamma_{1}\right)^{2}$.

we assume that $\lambda_{j}^{2}, \quad j=1,2,3$ are distinct and different from zero. We may assume without loss of generality that $\operatorname{Im} \lambda_{j}^{2}>0, \quad j=1,2,3$. (Mikelashvili [11]).

It is obvious that, from relation

\section{$D(\Delta) \boldsymbol{\Phi}=0$}

follows the following equations

$\Delta\left(\Delta+\lambda_{1}^{2}\right)\left(\Delta+\lambda_{2}^{2}\right)\left(\Delta+\lambda_{3}^{2}\right) \operatorname{div} \mathbf{u}=0$,

$\Delta\left(\Delta+\lambda_{1}^{2}\right)\left(\Delta+\lambda_{2}^{2}\right)\left(\Delta+\lambda_{3}^{2}\right) \varphi=0$,

$\Delta\left(\Delta+\lambda_{1}^{2}\right)\left(\Delta+\lambda_{2}^{2}\right)\left(\Delta+\lambda_{3}^{2}\right) p=0$,

$\Delta\left(\Delta+\lambda_{1}^{2}\right)\left(\Delta+\lambda_{2}^{2}\right)\left(\Delta+\lambda_{3}^{2}\right) \theta=0$.

Further, applying the operator $\Delta\left(\Delta+\lambda_{1}^{2}\right)\left(\Delta+\lambda_{2}^{2}\right)\left(\Delta+\lambda_{3}^{2}\right)$

to equation $(1)_{1}$ and using relations (4), we obtain

$\Delta \Delta\left(\Delta+\lambda_{1}^{2}\right)\left(\Delta+\lambda_{2}^{2}\right)\left(\Delta+\lambda_{3}^{2}\right) \mathbf{u}=0$

The prove is done.

Theorem 3. The regular solution $\mathbf{U}(\mathbf{u}, \varphi, p, \theta)$ of the system (1) can be represented as follows:
$\mathbf{u}=\boldsymbol{\Psi}-\operatorname{grad}\left[\left(m_{0}-1\right) h_{0}+\sum_{j=1}^{3} \frac{h_{j}}{\lambda_{j}^{2}}\right]$,

$\varphi=A_{0} h+\sum_{j=1}^{3} A h_{j}, \quad p=B_{0} h+\sum_{j=1}^{3} B_{j} h_{j}$

$\theta=C_{0} h+\sum_{j=1}^{3} C_{j} h_{j}$

where

$\operatorname{div} \mathbf{u}=h+\sum_{j-1}^{3} h_{j}, \quad \operatorname{div} \boldsymbol{\Psi}=m_{0} h, \quad \Delta h_{0}=h$,

$\Delta h=0, \quad\left(\Delta+\lambda_{j}^{2}\right) h_{j}=0$,

$A_{0} \delta_{0}=a\left(a_{0} b+m \beta\right)-b \gamma_{2}^{2}-m \gamma_{0} \gamma_{2}$

$-\beta \gamma_{2} \gamma_{1}+a_{0} \gamma_{0} \gamma_{1}$,

$B_{0} \delta_{0}=a\left(\alpha_{1} \beta-b m\right)+\beta \gamma_{1}^{2}-\alpha_{1} \gamma_{0} \gamma_{2}$

$+b \gamma_{2} \gamma_{1}-m \gamma_{0} \gamma_{1}$,

$C_{0} \delta_{0}=\gamma_{0}\left(\alpha_{1} a_{0}+m^{2}\right)-\gamma_{1}\left(m \beta+b a_{0}\right)$

$+\gamma_{2}\left(m b-\alpha_{1} \beta\right), \quad m_{0}=\frac{d_{3}}{\mu \delta_{0}}$,

$\delta_{j}=-\alpha k k_{0} \lambda_{j}^{6}+$

$\lambda_{j}^{4}\left[-\alpha_{1} k k_{0}+i \omega \alpha\left(a k T_{0}+a_{0} k_{0}\right)\right]+(i \omega)^{2} T_{0} \delta_{0}+$

$\lambda_{j}^{2} i \omega\left[\left(a_{0} \alpha_{1}+m^{2}\right) k_{0}+T_{0} k\left(\gamma_{1}^{2}+a \alpha_{1}\right)\right]+$

$\lambda_{j}^{2}(i \omega)^{2} \alpha T_{0}\left(\gamma_{2}^{2}-\alpha a_{0}\right)$,

$\delta_{0}=-a\left(\alpha_{1} a_{0}+m^{2}\right)+\alpha_{1} \gamma_{2}^{2}-a_{0} \gamma_{2}^{2}+2 m \gamma_{1} \gamma_{2}$,

$A_{j} \delta_{j}=b k k_{0} \lambda_{j}^{4}-\lambda_{j}^{2} i \omega\left[k_{0}\left(b a_{0}+\beta m\right)+k T_{0}\left(\gamma_{0} \gamma_{1}+a b\right]\right.$

$+(i \omega)^{2} T_{0} A_{0} \delta_{0}, \quad j=1,2,3$,

$B_{j} \delta_{j}=-i \omega \alpha \beta k_{0} \lambda_{j}^{4}+(i \omega)^{2} T_{0} B_{0} \delta_{0}-$

$\lambda_{j}^{2} i \omega\left[-i \omega \alpha T_{0}\left(\gamma_{0} \gamma_{2}-\alpha \beta\right)-k_{0}\left(\beta \alpha_{1}-b m\right)\right]$,

$C_{j} \delta_{j}=-i \omega \alpha T_{0} \gamma_{0} k \lambda_{j}^{4}+(i \omega)^{2} T_{0} C_{0} \delta_{0}$

$+\lambda_{j}^{2} i \omega T_{0}\left[-i \omega \alpha\left(\beta \gamma_{2}-a_{0} \gamma_{0}\right)+k\left(-\alpha_{1} \gamma_{0}+b \gamma_{1}\right)\right]$,

$\mu_{0}+b A_{0}-\beta B_{0}-\gamma_{0} C_{0}=\frac{d_{3}}{\delta_{0}}=\mu m_{0}$,

$b A_{j}-\beta B_{j}-\gamma_{0} C_{j}=-\mu_{0}$. 
Proof. It is well known, that the general solutions $\operatorname{div} \mathbf{u}, \varphi, p$ and $\theta$ of equations (4) can be written as follows (Vekua [28])

$\operatorname{div} \mathbf{u}=h+\sum_{j-1}^{3} h_{j}, \quad \varphi=A_{0} h+\sum_{j=1}^{3} A_{j} h_{j}$,

$p=B_{0} h+\sum_{j=1}^{3} B_{j} h_{j}, \quad \theta=C_{0} h+\sum_{j=1}^{3} C_{j} h_{j}$

It can be easily checked that; they are the solutions of Eqs. $(1)_{2},(1)_{3},(1)_{4}$. If supposing that $h$ and $h_{j}$ are known functions, taking into account the representations (8), to define the displacement vector $\mathbf{U}$ from Eq. $(1)_{1}$, we get the following non-homogeneous equation

$$
\Delta \mathbf{u}=-\operatorname{grad}\left[\left(m_{0}-1\right) h-\sum_{j=1}^{3} h_{j}\right]
$$

solution of which can be written as

$$
\mathbf{u}=\boldsymbol{\Psi}+\mathbf{u}_{0}
$$

where $\boldsymbol{\Psi}$ is an arbitrary harmonic function, $\mathbf{u}_{0}$

is a particular solution of equation (9).

$\mathbf{u}_{0}=-\operatorname{grad}\left[\left(m_{0}-1\right) h_{0}+\sum_{j=1}^{3} \frac{h_{j}}{\lambda_{j}^{2}}\right]$

Herein it is assumed that, the functions $\operatorname{div} \mathbf{u} \Psi, h_{j}$ and $h$ are interrelated by the following relations

$\operatorname{div} \mathbf{u}=h+\sum_{j-1}^{3} h_{j}, \quad \operatorname{div} \boldsymbol{\Psi}=m_{0} h$,

$\Delta h_{0}=h, \quad \Delta h=0, \quad \Delta \Psi=0$.

Thus, From the above reasoning we have obtained the general solution of the system (1) in the following form

$$
\begin{aligned}
& \mathbf{u}=\boldsymbol{\Psi}-\operatorname{grad}\left[\left(m_{0}-1\right) h_{0}+\sum_{j=1}^{3} \frac{h_{j}}{\lambda_{j}^{2}}\right], \\
& \varphi=A_{0} h+\sum_{j=1}^{3} A_{j} h_{j}, \quad p=B_{0} h+\sum_{j=1}^{3} B_{j} h_{j}, \\
& \theta=C_{0} h+\sum_{j=1}^{3} C_{j} h_{j},
\end{aligned}
$$

where $A_{j}, \quad B_{j}, C_{j}, j=0,1,2,3$ and $m_{0}$ are given by (7).

From (11) we conclude that the representation of a solution of $\mathbf{u}$ contains a harmonic, bi-harmonic, and a meta-harmonic functions, while the representations of $\varphi, \quad p$ and $\theta$ contains only a harmonic and a meta-harmonic functions.

\section{Explicit Solution of the Problem 1}

Let us introduce the spherical coordinates equalities $x_{1}=\rho \sin \xi \cos \eta, \quad x_{2}=\rho \sin \xi \sin \eta, \quad x_{3}=\rho \cos \xi$, $\rho=\sqrt{x_{1}^{2}+x_{2}^{2}+x_{3}^{2}}, \quad 0 \leq \eta \leq 2 \pi$. $y_{1}=R \sin \xi_{0} \cos \eta_{0}, \quad y_{2}=R \sin \xi_{0} \sin \eta_{0}$, $y_{3}=R \cos \xi_{0}$.

Taking into account the identity $(\mathbf{x} \cdot \operatorname{grad})=\rho \frac{\partial}{\partial \rho}$, by direct calculation, from (11) we obtain $(\mathbf{x} \cdot \mathbf{u})=(\mathbf{x} \cdot \Psi)-\rho \frac{\partial}{\partial \rho}\left[\left(m_{0}-1\right) h_{0}+\sum_{j=1}^{3} \frac{h_{j}}{\lambda_{j}^{2}}\right]$. $\sum_{k=1}^{3} \frac{\partial u_{k}}{\partial S_{k}}=\sum_{k=1}^{3} \frac{\partial \Psi_{k}}{\partial S_{k}}, \quad \frac{\partial}{\partial S_{k}}=[\mathbf{x} \cdot \nabla]$, $\nabla=\left(\frac{\partial}{\partial x_{1}}, \frac{\partial}{\partial x_{2}}, \frac{\partial}{\partial x_{3}}\right)$

It is easily to verified, that the function $(\mathbf{x} \cdot \Psi)$ satisfies the following equation $\Delta(\mathbf{x} \cdot \boldsymbol{\Psi})=2 \operatorname{div} \boldsymbol{\Psi}=2 m_{0} h$, the solution of which has the form

$(\mathbf{x} \cdot \Psi)=\Omega+2 m_{0} h_{0}$,

where $\Omega$ is an arbitrary harmonic function $\Delta \Omega=0$, the function $h_{0}$ is a bi-harmonic function and chosen such that $\Delta h_{0}=h, \quad \Delta h=0$.

Substituting the expression (13) into (12) and taking into account (11), we obtain

$$
\begin{aligned}
& (\mathbf{x} \cdot \mathbf{u})=\Omega+2 m_{0} h_{0}-\rho \frac{\partial}{\partial \rho}\left[\left(m_{0}-1\right) h_{0}+\sum_{j=1}^{3} \frac{h_{j}}{\lambda_{j}^{2}}\right] \\
& \varphi=A_{0} h+\sum_{j=1}^{3} A_{j} h_{j}, \quad p=B_{0} h+\sum_{j=1}^{3} B_{j} h_{j} \\
& \theta=C_{0} h+\sum_{j=1}^{3} C_{j} h_{j}, \quad \operatorname{div} \mathbf{u}=h+\sum_{j=1}^{3} h_{j} . \\
& \sum_{k=1}^{3} \frac{\partial u_{k}}{\partial S_{k}}=\sum_{k=1}^{3} \frac{\partial \Psi_{k}}{\partial S_{k}} .
\end{aligned}
$$

We are looking for a solution of the system (1), under boundary conditions of Problem 1, in the form (11), where the functions $h, h_{j}, \quad$ and $\Omega$ are sought in the form (Smirnov[27]) 
$h=\sum_{n=0}^{\infty}\left(\frac{\rho}{R}\right)^{n} Z_{n}(\xi, \eta), \quad \Omega=\sum_{n=0}^{\infty}\left(\frac{\rho}{R}\right)^{n} Y_{n}(\xi, \eta)$,

$h_{j}=\sum_{n=0}^{\infty} \Phi_{n}\left(\lambda_{j} \rho\right) Y_{j n}(\xi, \eta), \quad j=1,2,3$,

$\sum_{k=1}^{3} \frac{\partial \Psi_{k}}{\partial S_{k}}=\sum_{n=0}^{\infty}\left(\frac{\rho}{R}\right)^{n} Z_{n 1}(\xi, \eta), \quad \rho<R$,

$Z_{n}, \quad Y_{n}, Z_{n 1}$ and $Y_{j n}, \quad j=1,2,3$, are the unknown spherical harmonics of order $n$,

$\Phi_{n}\left(\lambda_{j} \rho\right)=\frac{\sqrt{R} J_{n+\frac{1}{2}}\left(\lambda_{j} \rho\right)}{\sqrt{\rho} J_{n+\frac{1}{2}}\left(\lambda_{j} R\right)}$,

$J_{n+\frac{1}{2}}\left(\lambda_{j} \rho\right)$ is the Bessel function.

Taking into account (15), we can write the particular solutions of equation $\Delta h_{0}=h$ in the form

$h_{0}=\frac{\rho^{2}}{2} \sum_{n=0}^{\infty} \frac{1}{3+2 n}\left(\frac{\rho}{R}\right)^{n} Z_{n}(\theta, \eta)$

For convenience we introduce the following functions:

$(\mathbf{x} \cdot \mathbf{f})^{+}=g_{1}^{+}, \quad(\operatorname{div} \mathbf{f})^{+}=g_{2}^{+}, \quad \sum_{k=1}^{3} \frac{\partial u_{k}}{\partial S_{k}}=g_{3}^{+}$,

$\varphi^{+}=g_{4}^{+}, \quad p^{+}=g_{5}^{+}, \quad \theta^{+}=g_{6}^{+}$.

We assume that the functions $g_{k}^{+}$can be represented in the form of series

$g_{k}^{+}=\sum_{n=0}^{\infty} g_{k n}^{+}, \quad k=1,2, \ldots, 6$

where $g_{k n}^{+}$are the spherical harmonic of order $\mathrm{n}$.

$g_{k n}^{+}=\frac{2 n+1}{4 \pi R^{2}} \iint_{S} P_{n}(\cos \gamma) g_{k}^{+} d S_{y}$

$P_{n}(\cos \gamma)$ is Legendre polynomial of the n-th order $\cos \gamma$ is an angle formed by the radius-vectors $O X$ and $O Y$

$\cos \gamma=\frac{1}{|\mathbf{x}||\mathbf{y}|} \sum_{k=1}^{3} x_{k} y_{k}$

$=\sin \xi \sin \xi_{0} \cos \left(\eta-\eta_{0}\right)+\cos \xi \cos \xi_{0}$.
Substituting the expressions (15) and (16) into (14), taking into account boundary conditions and passing to the limit as $\rho \rightarrow R$, for determining the unknown values we obtain the following system of equations:

$$
\begin{aligned}
& \Omega^{+}+2 m_{0} h_{0}-\rho \frac{\partial}{\partial \rho}\left[\left(m_{0}-1\right) h_{0}+\sum_{j=1}^{3} \frac{h_{j}}{\lambda_{j}^{2}}\right]=g_{1}^{+}, \\
& h^{+}+\sum_{j=1}^{3} h_{j}^{+}=g_{2}^{+}, \quad \sum_{k=1}^{3} \frac{\partial \Psi_{k}}{\partial S_{k}}=g_{3}^{+}, \\
& A_{0} h^{+}+\sum_{j=1}^{3} A_{j} h_{j}^{+}=g_{4}^{+}, \quad B_{0} h^{+}+\sum_{j=1}^{3} B_{j} h_{j}^{+}=g_{5}^{+}, \text {(18) } \\
& C_{0} h^{+}+\sum_{j=1}^{3} C_{j} h_{j}^{+}=g_{6}^{+} .
\end{aligned}
$$$$
\text { From }(18)_{3} \text { and }(18)_{4} \text { we get }
$$

$h^{+}(\xi, \eta)=$

$\frac{1}{m_{0} \mu}\left[\mu_{0} g_{2}^{+}+b g_{4}^{+}-\beta g_{5}^{+}-\gamma_{0} g_{6}^{+}\right]=G^{+}(\xi, \eta)$.

Let us consider the following system of quations

$\sum_{j=1}^{3} A_{j} h_{j}^{+}=g_{4}^{+}-A_{0} G^{+}=q_{1}$,

$\sum_{j=1}^{3} B_{j} h_{j}^{+}=g_{5}^{+}-B_{0} G^{+}=q_{2}$,

$\sum_{j=1}^{3} C_{j} h_{j}^{+}=g_{6}^{+}-C_{0} G^{+}=q_{3}$,

Following Theorem 1 we conclude that the determinant $d$ of system (20) is different from zero and the system (20) is uniquely solvable.

From (20) we find

$$
\begin{aligned}
& h_{1}^{+}=\frac{1}{d}\left[q_{1}\left(B_{2} C_{3}-B_{3} C_{2}\right)-q_{2}\left(A_{2} C_{3}-A_{3} C_{2}\right)\right. \\
& \left.+q_{3}\left(A_{2} B_{3}-A_{3} B_{2}\right)\right]=\mathrm{H}_{1}(\xi, \eta), \\
& h_{2}^{+}=\frac{1}{d}\left[-q_{1}\left(B_{1} C_{3}-B_{3} C_{1}\right)+q_{2}\left(A_{1} C_{3}-A_{3} C_{1}\right)\right. \\
& \left.-q_{3}\left(A_{1} B_{3}-A_{3} B_{1}\right)\right]=\mathrm{H}_{2}(\xi, \eta), \\
& h_{3}^{+}=\frac{1}{d}\left[q_{1}\left(B_{1} C_{2}-B_{2} C_{1}\right)-q_{2}\left(A_{1} C_{2}-A_{2} C_{1}\right)\right. \\
& \left.+q_{3}\left(A_{1} B_{2}-A_{2} B_{1}\right)\right]=\mathrm{H}_{3}(\xi, \eta),
\end{aligned}
$$




$$
d=\left|\begin{array}{lll}
A_{1} & A_{2} & A_{3} \\
B_{1} & B_{2} & B_{3} \\
C_{1} & C_{2} & C_{3}
\end{array}\right| \neq 0
$$

Thus the functions $h, h_{j}, \quad$ are known, from (18)we get

$\Omega^{+}=g_{1}^{+}-2 m_{0} h_{0}$

$+\rho \frac{\partial}{\partial \rho}\left[\left(m_{0}-1\right) h_{0}+\sum_{j=1}^{3} \frac{h_{j}}{\lambda_{j}^{2}}\right]=G_{4}^{+}$,

$\sum_{k=1}^{3} \frac{\partial \Psi_{k}}{\partial S_{k}}=g_{3}^{+}$

On the other hand, from (15), (19), (21) and (22), we get

$Z_{n}=G_{n}^{+}, \quad Y_{n}=G_{4 n}^{+}, \quad Y_{j n}=H_{j n}, \quad Z_{n 1}=g_{3 n}^{+}$,

where

$G_{n}^{+}(\xi, \eta)=\frac{2 n+1}{4 \pi R^{2}} \iint_{S} P_{n}(\cos \gamma) G^{+} d S_{y}$,

$g_{3 n}^{+}(\xi, \eta)=\frac{2 n+1}{4 \pi R^{2}} \iint_{S} P_{n}(\cos \gamma) g_{3}^{+} d S_{y}$,

$G_{4 n}^{+}(\xi, \eta)=\frac{2 n+1}{4 \pi R^{2}} \iint_{S} P_{n}(\cos \gamma) G_{4}^{+} d S_{y}$,

$H_{j n}(\xi, \eta)=\frac{2 n+1}{4 \pi R^{2}} \iint_{S} P_{n}(\cos \gamma) H_{j}^{+} d S_{y}$.

Through inserting the obtained values into (15), we obtain

$h(\mathbf{x})=\frac{1}{4 \pi R} \iint_{S} \frac{R^{2}-\rho^{2}}{|\mathbf{x}-\mathbf{y}|^{3}} G^{+}(y) d s$,

$\Omega(\mathbf{x})=\frac{1}{4 \pi R} \iint_{S} \frac{R^{2}-\rho^{2}}{|\mathbf{x}-\mathbf{y}|^{3}} G_{4}^{+}(y) d s$,

$\sum_{k=1}^{3} \frac{\partial \Psi_{k}}{\partial S_{k}}=\frac{1}{4 \pi R} \iint_{S} \frac{R^{2}-\rho^{2}}{|\mathbf{x}-\mathbf{y}|^{3}} g_{3}^{+}(y) d s$,

$h_{j}=\sum_{n=0}^{\infty} \Phi_{n}\left(\lambda_{j} \rho\right) H_{j n}(\xi, \eta), \quad j=1,2,3, \quad \rho<R$,

$h_{0}=\frac{\rho^{2}}{2} \sum_{n=0}^{\infty} \frac{1}{3+2 n}\left(\frac{\rho}{R}\right)^{n} G_{n}^{+}(\theta, \eta)$.

We assume that the functions $f_{k}^{+} \cdot k=1, \ldots, 5$, satisfy the following conditions on $S$ $f_{k}^{+} \in C^{5}(S), \quad k=1, \ldots, 6$.

Under these conditions the resulting series are absolutely and uniformly convergent.

\section{Explicit solution of the Problem 2}

Following the procedure, quite similarly as above, we can construct a solution of the Problem 2 for a thermoelastic porous space with a spherical cavity.

Note that the following identities are holds:

$$
\begin{aligned}
& \frac{\partial}{\partial n} \operatorname{grad} g=\frac{1}{\rho} \operatorname{grad}\left(\rho \frac{\partial}{\partial \rho}-1\right) g, \\
& \frac{\partial}{\partial n} \operatorname{rot} \mathbf{h}=\frac{1}{\rho} \operatorname{rot}\left[\left(\rho \frac{\partial}{\partial \rho}-1\right) \mathbf{h},\right.
\end{aligned}
$$

$\operatorname{div} \mathbf{n} g=\left(\frac{\partial}{\partial \rho}+\frac{1}{\rho}\right) g, \quad \frac{\partial}{\partial S_{k}} \frac{\partial}{\partial \rho}=\frac{\partial}{\partial \rho} \frac{\partial}{\partial S_{k}}$,

$\operatorname{div}[\mathbf{n} \cdot \operatorname{rot} \mathbf{u}]=-\frac{\partial}{\partial \rho} \operatorname{div} \boldsymbol{\Psi}$, if $\quad \Delta \boldsymbol{\Psi}=0$

$\lambda \operatorname{div} \mathbf{u}-b \varphi-\beta p-\gamma_{0} \theta=\mu\left(m_{0}-2\right) h-2 \mu \sum_{j=1}^{3} h_{j}$,

$\operatorname{div} \frac{\partial \mathbf{u}}{\partial n}=\left(\frac{\partial}{\partial \rho}+\frac{1}{\rho}\right) \operatorname{div} \mathbf{u}-\frac{1}{\rho}\left(\mathbf{n} \cdot \frac{\partial \mathbf{u}}{\partial n}\right)$,

$\left(\mathbf{n} \cdot \frac{\partial \mathbf{u}}{\partial n}\right)=\frac{1}{\rho}\left(\mathbf{x} \cdot \frac{\partial \Psi}{\partial n}\right)-\frac{\partial^{2}}{\partial \rho^{2}}\left[\left(m_{0}-1\right) h_{0}+\sum_{j=1}^{3} \frac{h_{j}}{\lambda_{j}^{2}}\right]$,

$\left(\mathbf{x} \cdot \frac{\partial \Psi}{\partial n}\right)=\frac{\partial(\mathbf{x} \cdot \Psi)}{\partial n}-\frac{(\mathbf{x} \cdot \Psi)}{\rho}, \quad(\mathbf{x} \cdot \operatorname{grad})=\rho \frac{\partial}{\partial \rho}$.

Taking into account these identities, let us rewrite the stress vector in the following form

$$
\begin{aligned}
& \mathbf{P}\left(\partial_{x}, \mathbf{n}\right) \mathbf{u}=2 \mu \frac{\partial \boldsymbol{\Psi}}{\partial n} \\
& -\frac{2 \mu}{\rho} \operatorname{grad}\left(\rho \frac{\partial}{\partial \rho}-1\right)\left[\left(m_{0}-1\right) h_{0}+\sum_{j=1}^{3} \frac{h_{j}}{\lambda_{j}^{2}}\right] \\
& +\mathbf{n}\left[\mu\left(m_{0}-2\right) h-2 \mu \sum_{j=1}^{3} h_{j}\right]+\frac{\mu}{\rho}[\mathbf{x} \cdot \operatorname{rot} \boldsymbol{\Psi}] .
\end{aligned}
$$




$$
\begin{aligned}
& (\mathbf{x} \cdot \mathbf{P U})=2 \mu\left(\mathbf{x} \cdot \frac{\partial \Psi}{\partial n}\right) \\
& -2 \mu \rho \frac{\partial^{2}}{\partial \rho^{2}}\left[\left(m_{0}-1\right) h_{0}+\sum_{j=1}^{3} \frac{h_{j}}{\lambda_{j}^{2}}\right] \\
& +\rho\left[\mu\left(m_{0}-2\right) h-2 \mu \sum_{j=1}^{3} h_{j}\right],
\end{aligned}
$$$$
\sum_{j=1}^{3} \frac{\partial}{\partial S_{k}} \frac{\partial(P u)_{k}}{\partial \rho}=2 \mu \frac{\partial}{\partial \rho} \sum_{j=1}^{3} \frac{\partial \Psi_{k}}{\partial S_{k}}
$$$$
\operatorname{div}\left(\mathbf{P}\left(\partial_{x}, \mathbf{n}\right) \mathbf{U}\right)=\frac{\mu m_{0} h}{\rho}-\frac{2 \mu}{\rho^{2}}\left(\mathbf{x} \cdot \frac{\partial \Psi}{\partial n}\right)
$$$$
+\frac{2 \mu}{\rho} \frac{\partial^{2}}{\partial \rho^{2}}\left[\left(m_{0}-1\right) h_{0}+\sum_{j=1}^{3} \frac{h_{j}}{\lambda_{j}^{2}}\right] \text {. }
$$

For convenience we introduce the following functions:

$$
\begin{aligned}
& (\mathbf{x} \cdot \mathbf{f})^{-}=g_{1}^{-}, \quad(\operatorname{div} \mathbf{f})^{-}=g_{2}^{-}, \\
& \left(\sum_{j=1}^{3} \frac{\partial(P u)_{k}}{\partial S_{k}}\right)^{-}=g_{3}^{-}, \\
& \left(\frac{\partial \varphi}{\partial n}\right)^{-}=g_{4}^{-}, \quad\left(\frac{\partial p}{\partial n}\right)^{-}=g_{5}^{-}, \quad\left(\frac{\partial \theta}{\partial n}\right)^{-}=g_{6}^{-} .
\end{aligned}
$$

By passing to the limit as $\rho \rightarrow R$, employing (24), and (11), we derive the following system of equations

$$
\begin{aligned}
& \left(\mathbf{x} \cdot \frac{\partial \Psi}{\partial n}\right)^{-}-R\left(\frac{\partial^{2}}{\partial \rho^{2}}\left[\left(m_{0}-1\right) h_{0}^{-}+\sum_{j=1}^{3} \frac{h_{j}^{-}}{\lambda_{j}^{2}}\right]\right)_{\rho=R} \\
& +\frac{R}{2}\left(\left(m_{0}-2\right) h^{-}-2 \sum_{j=1}^{3} h_{j}^{-}\right)_{\rho=R}=\frac{g_{1}^{-}}{2 \mu},
\end{aligned}
$$$$
2 \mu \frac{\partial}{\partial \rho} \sum_{j=1}^{3} \frac{\partial \Psi_{k}}{\partial S_{k}}=g_{3}^{-}
$$$$
m_{0} h-\frac{2}{R}\left(\left(\mathbf{x} \cdot \frac{\partial \Psi}{\partial n}\right)\right)
$$

$+2\left(\frac{\partial^{2}}{\partial \rho^{2}}\left[\left(m_{0}-1\right) h_{0}+\sum_{j=1}^{3} \frac{h_{j}}{\lambda_{j}^{2}}\right]\right)_{\rho=R}=\frac{R}{\mu} g_{2}^{-}$

$\left(m_{0}-1\right)\left(\frac{\partial h}{\partial n}\right)^{-}-\sum_{j=1}^{3}\left(\frac{\partial h_{j}}{\partial n}\right)^{-}=\frac{R}{2 \mu}\left(\frac{\partial}{\partial \rho}\left(g_{2}+\frac{g_{1}}{R^{2}}\right)\right)_{\rho=R}$,

$A_{0}\left(\frac{\partial h}{\partial n}\right)^{-}+\sum_{j=1}^{3} A_{j}\left(\frac{\partial h_{j}}{\partial n}\right)^{-}=g_{4}^{-}$

$B_{0}\left(\frac{\partial h}{\partial n}\right)^{-}+\sum_{j=1}^{3} B_{j}\left(\frac{\partial h_{j}}{\partial n}\right)^{-}=g_{5}^{-}$

$C_{0}\left(\frac{\partial h}{\partial n}\right)^{-}+\sum_{j=1}^{3} C_{j}\left(\frac{\partial h_{j}}{\partial n}\right)^{-}=g_{6}^{-}$

From here we get

$\left(\frac{\partial h}{\partial n}\right)^{-}=-\frac{\delta_{0}}{d_{3}(\lambda+\mu)}\left[-\mu_{0} \frac{R}{2 \mu}\left(g_{2}^{-}+\frac{g_{1}^{-}}{R^{2}}\right)+b g_{4}^{-}\right.$

$\left.-\beta g_{5}^{-}-\gamma_{0} g_{6}^{-}\right]=G^{-}$.

Let us consider the following system of equations

$\sum_{j=1}^{3} A_{j}\left(\frac{\partial h_{j}}{\partial n}\right)^{-}=g_{4}^{-}-A_{0} G^{-}=q_{1}$

$\sum_{j=1}^{3} B_{j}\left(\frac{\partial h_{j}}{\partial n}\right)^{-}=g_{5}^{-}-B_{0} G^{-}=q_{2}$,

$\sum_{j=1}^{3} C_{j}\left(\frac{\partial h_{j}}{\partial n}\right)^{-}=g_{6}^{-}-C_{0} G^{-}=q_{3}$.

(26)

Hence, following Theorem 1, we conclude, that the determinant of system (26) is different from zero. On solving the equations (26), similarly as above section, we get

$$
\begin{aligned}
& \left(\frac{\partial h_{1}}{\partial n}\right)^{-}=\frac{1}{d}\left[q_{1}\left(B_{2} C_{3}-B_{3} C_{2}\right)-q_{2}\left(A_{2} C_{3}-A_{3} C_{2}\right)\right. \\
& \left.+q_{3}\left(A_{2} B_{3}-A_{3} B_{2}\right)\right]=G_{1}^{-},
\end{aligned}
$$


$\left(\frac{\partial h_{2}}{\partial n}\right)^{-}=\frac{1}{d}\left[-q_{1}\left(B_{1} C_{3}-B_{3} C_{1}\right)+q_{2}\left(A_{1} C_{3}-A_{3} C_{1}\right)\right.$

$\left.-q_{3}\left(A_{1} B_{3}-A_{3} B_{1}\right)\right]=G_{2}^{-}$,

$\left(\frac{\partial h_{3}}{\partial n}\right)^{-}=\frac{1}{d}\left[q_{1}\left(B_{1} C_{2}-B_{2} C_{1}\right)-q_{2}\left(A_{1} C_{2}-A_{2} C_{1}\right)\right.$

$\left.+q_{3}\left(A_{1} B_{2}-A_{2} B_{1}\right)\right]=G_{3}^{-}$,

$\left(\mathbf{x} \cdot \frac{\partial \Psi}{\partial n}\right)^{-}=R\left(\frac{\partial^{2}}{\partial \rho^{2}}\left[\left(m_{0}-1\right) h_{0}^{-}+\sum_{j=1}^{3} \frac{h_{j}^{-}}{\lambda_{j}^{2}}\right]\right)_{\rho=R}$

$-\frac{R}{2}\left(\left(m_{0}-2\right) h^{-}-2 \sum_{j=1}^{3} h_{j}^{-}\right)_{\rho=R}+\frac{g_{1}^{-}}{2 \mu}=G_{4}^{-}$,

$2 \mu \frac{\partial}{\partial \rho} \sum_{j=1}^{3} \frac{\partial \Psi_{k}}{\partial S_{k}}=g_{3}^{-}$,

where

$\left(\mathbf{x} \cdot \frac{\partial \Psi}{\partial n}\right)=\left(\frac{\partial}{\partial \rho}-\frac{1}{\rho}\right)(\mathbf{x} \cdot \Psi)$,

$(\mathbf{x} \cdot \Psi)=\Omega+2 m_{0} h_{0}, \quad \Delta \Omega=0$.

We are looking for a solution of the system (1), under boundary conditions of Problem 2, in the form (11), where the functions $h, \quad h_{j}$ and $\sum_{k=1}^{3} \frac{\partial \Psi_{k}}{\partial S_{k}}$ are sought in the form (Vekua[28])

$h=-\sum_{n=0}^{\infty} \frac{R^{n+2}}{(n+1) \rho^{n+1}} Z_{n}(\xi, \eta), \quad \rho>R$,

(28)

$h_{j}=\sum_{n=0}^{\infty} \Phi_{n}\left(\lambda_{j} \rho\right) Y_{j n}(\xi, \eta), \quad j=1,2,3$,

$\sum_{k=1}^{3} \frac{\partial \Psi_{k}}{\partial S_{k}}=\sum_{n=0}^{\infty} \frac{R^{n+2}}{\rho^{n+1}} Z_{n 1}(\xi, \eta)$

$Z_{n}, Z_{n 1}$ and $Y_{j n}, \quad j=1,2,3$, are the unknown spherical harmonic of order $n$

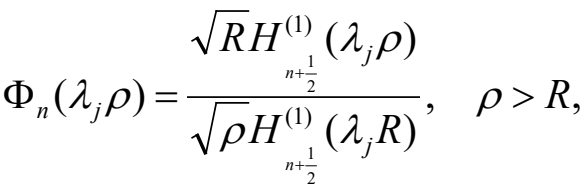

$H_{n+\frac{1}{2}}^{(1)}\left(\lambda_{j} \rho\right)$ is the Hankel function.
Taking into account (28), we can write the particular solutions of equation $\Delta h_{0}=h$ in the form

$h_{0}=\frac{\rho^{2}}{2} \sum_{n=0}^{\infty} \frac{1}{1-2 n} \frac{R^{n+2}}{\rho^{n+1}} Z_{n}(\xi, \eta)$

On the other hand, from $(25)_{a}$, (27) and (28), we get

$Z_{n}(\xi, \eta)=G_{n}^{-}, \quad \rho>R, \quad-2 \mu(n+1) Z_{n 1}=g_{3 n}^{-}$,

$Y_{j n}(\xi, \eta)=\frac{G_{j n}^{-}}{\omega_{n}\left(\lambda_{j} R\right)}, \quad j=1,2,3$,

$\omega_{n}\left(\lambda_{j} R\right)=\left(\frac{\partial}{\partial \rho} \Phi_{n}\left(\lambda_{j} \rho\right)\right)_{\rho=R}$,

where $G_{n}^{-}, \quad g_{3 n}^{-}$and $G_{j n}^{-}$are the spherical harmonics of order n

$G^{-}=\sum_{n=0}^{\infty} G_{n}^{-}, \quad G_{j}^{-}=\sum_{n=0}^{\infty} G_{j n}^{-}, \quad g_{3}^{-}=\sum_{n=0}^{\infty} g_{3 n}^{-}$,

$G_{n}^{-}=\frac{2 n+1}{4 \pi R^{2}} \iint_{S} P_{n}(\cos \gamma) G^{-}\left(\xi_{0}, \eta_{0}\right) d S_{y}$,

$G_{j n}^{-}=\frac{2 n+1}{4 \pi R^{2}} \iint_{S} P_{n}(\cos \gamma) G_{j}^{-}\left(\xi_{0}, \eta_{0}\right) d S_{y}$,

$g_{3 n}^{-}=\frac{2 n+1}{4 \pi R^{2}} \iint_{S} P_{n}(\cos \gamma) g_{3}^{-}\left(\xi_{0}, \eta_{0}\right) d S_{y}$.

Using (29) and (28), we obtain

$h=-\sum_{n=0}^{\infty} \frac{R^{n+2}}{(n+1) \rho^{n+1}} G_{n}(\xi, \eta)$,

$h_{j}=\sum_{n=0}^{\infty} \frac{\Phi_{n}\left(\lambda_{j} \rho\right)}{\omega\left(\lambda_{j} R\right)} G_{j n}^{-}\left(\xi_{0}, \eta_{0}\right), \quad \rho>R$,

$\sum_{k=1}^{3} \frac{\partial \Psi_{k}}{\partial S_{k}}=-\frac{1}{2 \mu} \sum_{n=0}^{\infty} \frac{R^{n+2}}{\rho^{n+1}} \frac{g_{3 n}^{-}}{n+1}$

$h_{0}=\frac{\rho^{2}}{2} \sum_{n=0}^{\infty} \frac{1}{1-2 n} \frac{R^{n+2}}{\rho^{n+1}} G_{n}(\xi, \eta)$.

Since the functions $h, \quad h_{j}$ and $h_{0}$ are known, to determination of $\Omega$, from (27), when $\rho=R$, we get 
$\left(\frac{\partial \Omega}{\partial n}-\frac{\Omega}{R}\right)^{-}=G_{4}^{-}-2 m_{0}\left(\frac{\partial}{\partial \rho}-\frac{1}{R}\right) h_{0}=G_{5}^{-}$.

Thus, we have obtained for the Laplace equation

$\Delta \Omega=0, \quad x \in D^{-}$,

the Robin boundary value problem

$\left(\frac{\partial \Omega}{\partial n}-\frac{\Omega}{R}\right)^{-}=G_{5}^{-}$,

the solution of which has the form

$\Omega(\mathbf{x})=\frac{1}{2 \pi} \iint_{S} \frac{g(y)}{r(x, y)} d s$,

where $g(y)$ is a solution of the following Fredholm integral equation of second kind

$-g(x)+\frac{1}{2 \pi} \iint_{S} \frac{\partial}{\partial n_{x}} \frac{1}{r(x, y)} g(y) d s$

$-\frac{1}{2 \pi R} \iint_{S} \frac{g(y)}{r(x, y)} d s=G_{5}^{-}$,

$r^{2}(x, y)=\sum_{j=1}^{3}\left(x_{j}-y_{j}\right)^{2}$

It is well known that integral equation (31) is always solvable.

Substituting the obtained values into (28) and (11), we get the final form for solution of the considered Problem 2.

We assume that the functions $f_{k}^{-}$satisfy the following conditions on $S$

$f_{k}^{-} \in C^{5}(S), \quad j=1,2, \ldots, 6$

Under these conditions the resulting series are absolutely and uniformly convergent. Moreover we assume that the functions $\hat{\mathbf{G}}^{-}$

, $\hat{\mathbf{G}}_{k}^{-}$and its first order derivatives are absolutely integrable and vanishing at infinity functions.

Thus, the considered problems are completely solved.

\section{Conclusions}

The main results of this work can be formulated as follows:

- The general solution of the system of equations in the considered theory is presented by means of elementary (harmonic, metaharmonic and bi-harmonic) functions;

- Explicit solutions of problems for a sphere and for a space with spherical cavity is presented. The obtained solutions are given by means of the harmonic, bi-harmonic and meta-harmonic functions. For the harmonic functions the Poisson tyr formulas are obtained. The bi-harmonic and meta-harmonic functions are presented as absolutely and uniformly convergent series. The harmonic function $\Omega(\mathbf{x})$ is given by (30), where the function $g(y)$ is a solution of the Fredholm integral equation of second kind.

\section{Declaration of Conflict of Interests}

The author declares that there is no conflict of interest. They have no known competing financial interests or personal relationships that could have appeared to influence the work reported in this paper.

\section{References}

[1.] Nunziato, J.W., and Cowin, S.C., A nonlinear theory of elastic materials with voids. Arch. Rational Mech. Anal 72(2) (1979) 175201

[2.] Cowin, S.C. and Nunziato, J.W., Linear theory of elastic materials with voids. J. Elasticity 13(2) (1983) 125-147.

[3.] De Boer, R. Theory of porous media. Highlights in the historical development and current state. Berlin-Heidelberg-New York: Springer, 2000.

[4.] Straughan, B., Mathematical aspects of multi-porosity continua. Advances in Mechanics and Mathematics. 38: Springer, Switzerland, 2017.

[5.] Straughan, B., Stability and wave motion in porous media. New York: Springer, 2008.

[6.] Svanadze, M., Potential method in mathematical theories of multi-porosity media. Basel: Springer, 2019.

[7.] Ieşan, D., Quintanilla, R., On a theory of thermoelastic materials with a double porosity structure. J. Thermal Stresses 37 (2014) 1017-1036.

[8.] Svanadze, M., Steady vibration problems in the coupled linear theory of porous elastic solids. Math. Mech. of solids 25(3) (2020) 768-790.

[9.] Svanadze, M., Boundary integral equations method in the coupled theory of thermoelasticity for porous materials. Proceedings of the ASME 2019 International Mechanical Engineering Congress and Exposition. Volume 9: Mechanics of Solids, Structures, and Fluids. Salt Lake City, Utah, USA. November 11-14, 2019. V009T11A033. ASME.

[10.] Mikelashvili, M., Quasi-static problems in the coupled linear theory of elasticity for porous materials. Acta Mech 231(3) (2020) 877-897.

[11.] Mikelashvili, M., Quasi-static problems in the coupled linear theory of thermoelasticity. J. Thermal Stresses 44(2) (2021) 236259 .

[12.] Ciarletta, M., Scalia, A., On uniqueness and reciprocity in linear thermoelasticity of materials with void. J. Elasticity 32(1993) 117.

[13.] Bitsadze, L., Explicit solution of the Dirichlet boundary value problem of elasticity for porous infinite strip. Z. Angew. Math. Phys 71(5)2020) 145. 
[14.] Bitsadze, L., Tsagareli, I., The solution of the Dirichlet BVP in the fully coupled theory for spherical layer with double porosity. Meccanica 51(2016) 1457-1463.

[15.] Jaiani, G., Bitsadze, L., Basic Problems of Thermoelasticity with microtemperatures in the half-space. J. Thermal Stresses 41(9) (2018) 1101-1114.

[16.] Bitsadze, L., Zirakashvili, N., Explicit solutions of the boundary value problems for an ellipse with double porosity. Advances in Mathematical Physics 2016; Article ID 1810795 DOI:10.1155/2016/1810795.

[17.] Bitsadze, L. Explicit solutions of boundary value problems of elasticity for circle with a double voids. J. Braz. Soc. Mech. Sci. Eng 41(2019) 383.

[18.] Bitsadze, L., On one BVP for a thermo-microstretch elastic space with spherical cavity. Turk. J. Math 42(5) (2018) 2101 -2111.

[19.] Bitsadze, L., Explicit solutions of the BVPs of the theory of thermoelasticity for an elastic circle with voids and microtemperatures. J. of Applied Mathematics and Mechanics 100(10) (2020).

[20.] Singh, B., Raj Pal., Surface waves Propagation in a generalized theroelastic material with voids. Applied Mathematics 2(2011) 521-526.

[21.] Pompei, A. and Scalia, A., On a steady vibrations of elastic materials with voids. J. of Elasticity 36(1994) 1-26.

[22.] Magaa, A., Quintanilla, R. On the spatial behavior of solutions for porous elastic solids quasi-static microvoids. Mathematical and Computer modeling 44(2006) 710- 716.

[23.] Coussy, O., Mechanics and Physics of Porous Media. Dordrecht: Springer, 2005.

[24.] Svanadze, M., and De Cicco, S., Fundamental solutions in the full coupled linear theory of elasticity for solids with double porosity. Arch. Mech 65(5) (2013) 367-390.

[25.] Straughan, B. Modelling questions in multi-porosity elasticity. Meccanica 51(2016) 2957-2966.

[26.] Svanadze, M.Steady vibration problems in theory of elasticity for materials with double woids.Acta Mech 229(4)(2018) 1517-1536.

[27.] Smirnov, V.I., Course of Higher Mathematics. v. III, part 2, Moscow: Nauka, 1969.

[28.] Vekua, I., New methods for solving elliptic equations. Amsterdam, New-York, Oxford, North-Holland Publ. Company 1967.

\section{How to Cite This Article}

Bitsadze, L., Boundary Value Problems of Thermoelasticity for Porous Sphere and for A Space with Spherical Cavity, Brilliant Engineering, 1(2022), 1-10. https://doi.org/10.36937/ben.2021.4501 